\title{
UMA ANÁLISE DO DISCURSO DA DIMENSÃO CULTURAL DA ISKO
}

\author{
Isadora Victorino Evangelista* \\ Thiago Henrique Bragato Barros \\ João Batista Ernesto de Moraes
}

RESUMO

Palavras-chave: Organização e Representação do Conhecimento. Aspectos
socioculturais em ORC. International Society for Knowledge

Palavras-chave: Organização e Representação do Conhecimento. Aspectos
socioculturais em ORC. International Society for Knowledge Organization. Análise do Discurso.

Considerando o crescimento das pesquisas sobre as questões culturais em Organização e Representação do Conhecimento (ORC) e a International Society for Knowledge Organization (ISKO) como a instituição mais representativa na divulgação de pesquisas em ORC, questiona-se em que medida os aspectos culturais da área apareceram e foram construídos nos eventos internacionais da referida sociedade. Para tanto, objetiva-se analisar os conceitos relacionados a essa temática por meio dos editoriais publicados nos anais dos eventos realizados. Como metodologia, utilizou-se a Análise do Discurso Foucaultiana estudada por Pêcheux e seu grupo, por se tratar de uma metodologia que possui como característica a ruptura de ideologias e que preza por evidenciar relações entre dominados e dominantes. Foi possível observar que os conceitos culturais foram mencionados apenas no $4^{\circ}$ Congresso realizado pela sociedade, em que se refletiu uma preocupação com as questões multiculturais e multilinguísticas. Após esse primeiro momento, essas questões apareceram em subtemas, temas de congressos, mesas-redondas e no último congresso realizado, houve uma dimensão dedicada somente a essas questões. Os resultados permitiram inferir que a tônica sociocultural vem se apresentando e ganhando importante espaço nas últimas pesquisas sobre o campo, demonstrando que não apenas é necessária para o desenvolvimento ético dos processos de organização, mas que também é fundamental que pesquisas nesse sentido se façam cada vez mais presentes.

\footnotetext{
* Mestre em Ciência da Informação pela Universidade Estadual Paulista Julio de Mesquita Filho, Brasil. Doutoranda em Ciência da Informação pela Universidade Estadual Paulista Julio de Mesquita Filho, Brasil. Pesquisa com apoio da Fundação de Amparo à Pesquisa do Estado de São Paulo, Brasil. Brasil.

E-mail: isadora.biblio@marilia.unesp.br.

** Doutor em Ciência da Informação pela Universidade Estadual Paulista Júlio de Mesquita Filho, Brasil. Docente permanente no Programa de PósGraduação em Ciência da Informação da Universidade Federal do Pará, Brasil. E-mail: sean.vogel@gmail.com.

**** Doutor em Estudos Literários pela Universidade Estadual Paulista Júlio de Mesquita Filho, Brasil. Docente permanente no Programa de Pós-Graduação em Ciência da Informação da Universidade Estadual Paulista Júlio de Mesquita Filho, Brasil.

E-mail: jota@marilia.unesp.br.
}

\section{INTRODUÇÃO}

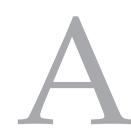

Organização

e Representação

do

Conhecimento (ORC) é considerada um

dos principais campos de estudo em Ciência da Informação, tendo na International Society for Knowledge Organization (ISKO) um importante veículo de produção e divulgação de pesquisas que trabalham com os temas relacionados ao referido campo.

Embora os aspectos socioculturais dos processos e ferramentas da Organização e
Representação do Conhecimento tenham ganhado espaço e demonstrado sua importância no ambiente científico ao longo dos últimos anos, ainda não se tinha certo em que medida isso foi construído na instituição ISKO Internacional.

A presente pesquisa identifica como problemática a investigação sobre como ocorre a construção dos conceitos culturais e da preocupação ética na ORC durante a trajetória de crescimento e divulgação da ISKO Internacional. Para tanto, objetivou-se analisar os conceitos relacionados à essa temática, por meio não dos 
artigos publicados, como normalmente ocorre, mas principalmente por meio de seus editoriais, por representarem de forma clara a linha científica e visões de mundo da instituição.

Como método de análise, aplicou-se a Análise do Discurso de origem francesa, estudada por Pêcheux e seu grupo, a partir das obras de Michel Foucault. Como esse tipo de análise pressupõe a materialidade do objeto de pesquisa, recorreu-se aos editoriais publicados e registrados nos anais do evento. A escolha da metodologia se justifica por se tratar de uma análise que preza por dar destaque e visibilidade às relações entre dominados e dominantes, muitas vezes presentes em estudos socioculturais.

Como justificativa científica da presente pesquisa, têm-se a divulgação da área de estudos dos aspectos sociais e culturais da Organização e Representação do Conhecimento, área essa que está em constante crescimento e buscando sua sedimentação no campo cientifico, além de demonstrar como ocorreram rupturas e como as pesquisas nesse âmbito foram tomando seu espaço no decorrer dos últimos anos.

A primeira sessão trata dos aspectos teóricos que envolvem a Organização e Representação do Conhecimento, além de tratar sobre os objetivos e o surgimento da International Society for Knowledge Organization. A segunda sessão apresentou a metodologia utilizada, aprofundando-se em seus aspectos históricos e conceituais. A próxima sessão expôs-se os resultados a que a pesquisa chegou, a que se segue as considerações finais do presente artigo.

\section{PRESSUPOSTOS TEÓRICOS}

Segundo Hjorland (2016), a Organização e Representação do Conhecimento é um dos campos de estudo da Ciência da Informação que trata sobre a descrição, representação e registro de documentos ou representação de documentos e que pode ser realizada por meios intelectuais (humanos) ou racionais (processos computacionais). Segundo o autor, esse campo pode ser dividido em sistemas de ORC e processos de ORC - o primeiro diz respeito aos sistemas de classificação, tesauros, listas de cabeçalho de assunto, entre outros e o segundo, se caracteriza pelos processos de indexação, classificação e catalogação.
Atualmente, a maior associação a nível internacional que trabalha com o estudo da Organização e Representação do Conhecimento no âmbito da Ciência da Informação, é a International Society for Knowledge Organization. A fundação foi criada em 1989, por Ingetraut Dahlberg na Alemanha, a partir da Society for Classification.

A ISKO possui por objetivos promover a pesquisa e desenvolvimento de sistemas de organização do conhecimento; proporcionar a comunicação e divulgação das pesquisas na área para seus membros e agir como um ponto em comum entre as instituições e agências que trabalham em função do avanço dos estudos em organização do conhecimento ${ }^{1}$.

A sociedade ainda possui uma série de veículos de comunicação científica, como: os eventos internacionais, que acontecem a cada dois anos; os capítulos nacionais e regionais que também são bienais e estão divididos em 14 grupos (Brasil; Canadá e Estados Unidos; China; França; Alemanha, Áustria e Suíça; Índia; Irã; Itália; Tunísia, Argélia e Marrocos; Polônia; Cingapura; Espanha e Portugal; Reino Unido e África Ocidental, a ser implantado ainda este ano); e ainda, a revista Knowledge Organization, que possui conceito A1 pelo Qualis da CAPES.

A dimensão social e cultural da Organização e Representação do Conhecimento tem sido representada no âmbito das publicações da ISKO, principalmente por meio dos temas de suas conferências internacionais, em especial os eventos realizados nos anos de 2002 (Seventh International ISKO Conference, realizada em Granada), que teve como tema Challenges in knowledge representation and organization for the 21st century; no ano de 2008 (Tenth International ISKO Conference, realizado em Montréal), com o tema Culture and identity in knowledge organization e a conferência mais recente, a de 2016 (Fourteenth International ISKO Conference, realizada no Rio de Janeiro), com o tema Knowledge organization for a sustainable world: challenges and perspectives for cultural, scientific, and technological sharing in a connected society.

As pesquisas nessa dimensão tem ainda seu destaque por meio de pesquisas

\footnotetext{
I Disponível em http://www.isko.org/about.html.
} 
como Guimarães e Fernández-Molina, que analisam 16 códigos de ética do profissional bibliotecário, para identificar um grupo de valores comuns a essa profissão; GarcíaGutiérrez (2002) apresenta o conceito de ética transcultural de mediação, em que a diversidade cultural deve ser refletida no âmbito da organização do conhecimento; López-Huertas (2008) que considera serem os estudos éticos em Organização e Representação do Conhecimento uma grande tendência e afirma a necessidade de seu aprofundamento; Guimarães et al. (2008) identificam um grupo de valores e problemas intrínsecos ao profissional da Organização e Representação do Conhecimento, dentre outros.

É possível perceber que a ISKO tem sido um ambiente propício para o desenvolvimento de pesquisas sobre os estudos éticos em ORC, com trabalhos que refletem e criticam práticas tradicionais e visões positivistas do profissional da Ciência da Informação. Esse fator aliado à importância que a ISKO atualmente possui no âmbito da ORC, demonstra que as pesquisas na dimensão cultural do campo têm revelado sua importância e pertinência ao longo das últimas décadas.

\section{METODOLOGIA}

A metodologia utilizada na pesquisa foi a Análise do Discurso (AD) de linha francesa, baseada nos trabalhos de Michel Foucault e estudada por Pêcheux e seu grupo. A análise possui por tríade a língua, o discurso e a ideologia; se utilizando da estrutura linguística para identificar questões ideológicas, considerando a trajetória do documento, sua historicidade; não apenas o que é dito, mas como é dito.

No início do século XX, os estudos linguísticos baseavam-se em uma lógica quase matemática, centrados nos aspectos da língua/fala, sem considerar o contexto em que são produzidos. Em 1916 então, Saussure publica o Curso de Linguística Geral, que dá início à Linguística Moderna, onde expõe o estruturalismo e sistematicidade dos estudos linguísticos, como uma dicotomia composta por um signo. Pela teoria saussuriana, o signo é composto pelo significante (imagem acústica) e pelo significado (conceito), indo além do que até então era compreendido no âmbito linguístico (BARROS, 2015).

A AD alia então, o conceito de Linguística estrutural conceituada por Saussure, com outros dois grandes autores das Ciências Humanas: Freud, dando base teórica para as interpretações do subconsciente e Marx, com suas ideias relativas às questões econômicas; revelando-se assim, como uma resposta da Linguística para questões até então consideradas como fora de seu domínio e buscando revelar elementos históricos e ideológicos por meio de extratos textuais (BARROS, 2015).

Segundo Barros (2015), por meio da ideologia, é possível identificar por exemplo, lutas de classes, relações dominantes, aspectos "apagados" propositalmente, dentre outros. A ideologia funciona como uma lente, um modo de visão sob determinado aspecto. Para a Análise do Discurso, a noção de ideologia encontra-se muito próxima do conceito de sujeito, pois todo sujeito possui uma ideologia e o discurso "[...] é uma instância da linguagem em que a ideologia se inscreve" (BARROS, 2015, p. 46). Só é possível evidenciar essa ideologia e discurso, por meio do conflito e da contradição.

Segundo Mittmnn (2007), a Análise do Discurso possui como compromisso, não permitir que discursos com posicionamentos elitistas e mercantis, sejam copiados e propagados sem nenhuma reflexão, de forma a não confirmar consensos objetivos e já legitimados, mas sim, expor aquilo que é imposto por meio de processos discursivos.

Pode-se perceber que os estudos em $\mathrm{AD}$, visam não só a compreensão formalista da língua, mas a língua em uso. Por meio dessa metodologia, é possível refletir não apenas sobre os estudos de linguagem, mas sobre esses estudos em relação à sociedade.

Ainda segundo Barros (2015), a AD possui três momentos distintos e importantes. O primeiro, tem seu início em 1968, em que Pêcheux fundamenta a Análise do Discurso baseando-se na Análise Automática do Discurso, que tem por objeto textos políticos e doutrinários, onde a ideologia encontra-se mais evidente $\mathrm{e}$, nesse primeiro momento, a $\mathrm{AD}$ tem passos metodológicos fixos e definidos etapa por etapa. É também nesse período que é publicado na revista Languages um artigo denominado 
Discourse Analysis, por Z. S. Harris, em que se revela uma maior preocupação estrutural, não semântica.

No segundo momento, Pêcheux se aproxima das obras de Foucault e as relaciona com a AD, embora a luta de classes ainda seja determinante, outros fatores referentes à relações ideológicas têm seu início.

Apesar de nunca ter sido a intenção de Foucault, obras como "Arqueologia do Saber", "A ordem do discurso" e "As palavras e as coisas" serviram de base para os estudos em AD. Segundo Gregolin (2006), Foucault em suas obras busca construir a história do homem perante o sistema, que o entende como objeto, revelando um conjunto de opiniões simultâneas e contraditórias. Para tanto, o autor possui como foco de investigação a relação entre a História, o discurso, o sujeito e a produção de sentidos.

Como metodologia de análise, Foucault busca então estabelecer um método arqueológico, um método de "escavação" que permite analisar relações entre discursos e práticas dominantes, em discursos consolidados e registrados. Esse método envolve a busca e a exposição desses discursos, de maneira a evidenciar elementos e práticas discursivas que associadas esclarecem a maneira de pensar de uma determinada época. Para o autor, não há um princípio de continuidade nesses discursos, pelo contrário, os discursos de diferentes épocas não possuem sentido de progressão (GREGOLIN, 2006).

Outro conceito definido por Foucault, é a ideia de acontecimento discursivo, ou seja, analisar um conjunto de enunciados finito, limitado e materializado, de maneira a entender as circunstâncias que possibilitaram sua utilização em determinado período histórico. Dessa forma, é possível identificar fatos discursivos que antecedem um evento, mas que podem o explicar ou até mesmo, o determinar. A ideia de descontinuidade está bem implícita aqui, diferentemente do que até então era idealizado pela História tradicional, que prezava por certa linearidade (GREGOLIN, 2006).

Os enunciados devem ser entendidos enquanto a própria função enunciativa, que é definida por Gregolin (2006, p. 89) como elemento "[...] produzido por um sujeito em um lugar institucional, determinado por regras sóciohistóricas que definem e possibilitam que ele seja enunciado". Para a autora, em um enunciado não deve ser levado em conta apenas as funções gramaticais, mas todo a conjuntura que envolve o sujeito e a História.

Deve-se ainda levar em consideração o conceito de formação discursiva, que é constituída a partir de grupos de enunciados. Quando se analisa uma formação discursiva, chega-se a uma certa ideia de positividade, onde todos os discursos possuem a ideia da verdade e concordam entre si (GREGOLIN, 2006).

Para que se instaure o que irá pertencer a uma Formação Discursiva, Mittmann (2007) sugere que o analista reflita sobre a teorização, por meio de quadro epistemológico, e estabeleça as relações entre os grupos de enunciados.

Tem-se ainda a noção de práticas discursivas, que são os preceitos, ainda que não formalizados, existentes em determinado período histórico que definem o desenvolvimento daquela sociedade e dos discursos produzidos por ela. O espaço em que o discurso é consentido, as relações entre os sujeitos coordenados e subordinados e apropriação desses discursos são o que compõe a noção de práticas discursivas (GREGOLIN, 2006).

Quando reunimos os conceitos de acontecimento discursivo, enunciados, formação discursiva, prática discursiva, dentre outros, chegamos então à concepção de arquivo, que é o conjunto de todos os elementos que irão compor a análise (GREGOLIN, 2006)

$\mathrm{O}$ controle de todos esses elementos relacionados ao discurso, ou até mesmo do próprio discurso, é regulamentado pelo autor, que claro, não deve ser entendido como um único indivíduo, mas como o grupo de indivíduos que se utilizam desses discursos. Segundo Gregolin (2006), Foucault propõe quatro particularidades do autor: ele está ligado a um sistema jurídico e institucional; essa função não é uniforme em todos os discursos, épocas ou civilizações; não é exercida arbitrariamente, mas por meio de uma série de operações específicas e complexas e não deve ser entendida como um único indivíduo.

Além da noção de autor, outros dois sistemas de controle do discurso podem ser identificados: as disciplinas, que definem quais enunciados serão julgados como verdadeiros e quais serão tidos como falsos, e ainda, as 
doutrinas, que limitam quais sujeitos irão ter acesso aos discursos. Esses controles são exercidos por meio, por exemplo, das instituições religiosas, do sistema educacional, dentre outros (GREGOLING, 2006).

Na década de 80, a Análise do Discurso inicia uma nova fase, em que a análise passa de ser exclusivamente em expressões textuais, para outros elementos passíveis de estudo, como por exemplo imagens e discursos orais. Nesse momento, o grupo de Pêcheux se aproxima de textos de Michael Bahktin, entendendo a $\mathrm{AD}$ como uma área de estudo semiológico e estabelecendo termos como memória discursiva e interdiscursividade, que considera as redes de sentido entre os enunciados e que pode remeter a diferentes enunciados anteriores, ao invés de termos como formação discursiva (BARROS, 2015; FREITAS, 2010).

A partir desse terceiro momento, é possível distinguir duas correntes teóricas da Análise do Discurso: a corrente francesa, que tem por objeto de estudo os discursos escritos e construídos, com um maior nível de formalização e a anglo-saxã, que investiga os discursos orais e cotidianos, relacionados a temas em que aplicação ideológica é mais evidenciada, como violência sexual e racismo (BARROS, 2015).

É importante ressaltar que a Análise do Discurso deve ser entendida enquanto um movimento de ruptura, não de continuidade, um deslocamento no âmbito da teoria das ciências humanas e sociais, agindo como um entremeio para a instauração de uma nova região disciplinar (BARROS, 2015).

A Análise do Discurso se instaura então, enquanto uma metodologia que se baseia em textos recuperados por meio de palavraschave, que referem-se a determinado tema a ser estudado e que estejam devidamente registrados para que componham o corpus de análise. A análise será realizada por etapas e levará em consideração a memória discursiva dos produtores e usuários dos objetos analisados.

No Brasil, importantes Programas de Pós-Graduação têm se dedicado ao estudo da Análise do Discurso, sendo o precedente deles o Programa de Pós-Graduação em Linguística da UNICAMP, que abriu espaço para outros, como o da Universidade Federal do Rio Grande do Sul e da Universidade de São Paulo.
Na Ciência da Informação, colaborações têm sido feitas por Frohmann (1994, 2001). Para o autor, a análise do discurso pode ser considerada um importante método para entender questões da área, em especial no âmbito dos estudos sociais e culturais em Ciência da Informação, pois permite analisar como e em quais conjunturas importantes teóricos constroem ideias sobre conceitos da área. Para tanto, o autor analisa o discurso dos trabalhos de Dewey, que tem seu surgimento em meio a uma visão tecnoburocrata, que buscava a racionalização e a especialização; em outra análise, Frohmann interpreta a visão de documentação para Otlet, por meio da perspectiva foucaultiana de discurso, identificando assim que ambos possuem o caráter social e materializado da informação como base.

No âmbito nacional, há importantes contribuições, como a de Lídia Freitas (2003, 2004, 2010), que busca apresentar como se construiu e a divulgar a análise do discurso em CI; Nadea Gaspar $(2008,2010)$, que se utiliza da análise do discurso em recursos audiovisuais e imagéticos; e Thiago Barros (2011, 2013, 2014), que estuda a AD no âmbito da arquivística.

A presente pesquisa buscou então, analisar de maneira discursiva, como os conceitos relacionados aos aspectos sociais e culturais da Organização e Representação do Conhecimento surgiram e se desenvolveram no domínio da International Society for Knowledge Organization.

Para tanto, foram analisados os prefácios e introduções - quando presentes - dos anais dos 14 eventos internacionais realizados pela referida sociedade, sendo eles:

- 1990, realizado em Darmstadt, na Alemanha, com o tema Tools for knowledge organization and the human interface;

- $\quad$ 1992, realizado em Madras, na Índia, com o tema Cognitive paradigms in knowledge organisation;

- 1994, realizado em Copenhagen, na Dinamarca, com o tema Knowledge organization and quality management;

- 1996, realizado em Washington, nos Estados Unidos, com o tema Knowledge organization and change;

- $\quad$ 1998, realizado em Lille, na França, com o tema Structures and relations in knowledge organization; 
- 2000, realizado em Toronto, no Canadá, com o tema Dynamism and stability in knowledge organization;

- $\quad 2002$, realizado em Granada, na Espanha, com o tema Challenges in knowledge representation and organization for the 21st century;

- 2004, realizado em Londres, na Inglaterra, com o tema Knowledge organization and the global information society;

- $\quad$ 2006, realizado em Viena, na Áustria, com o tema Knowledge organization for a global learning society;

- 2008, realizado em Montréal, no Canadá, com o tema Culture and identity in knowledge organization;

- 2010, realizado em Roma, na Itália, com o tema Paradigms and conceptual systems in knowledge organization;

- $\quad$ 2012, realizado em Mysore, na Índia, com o tema Categories, contexts and relations in knowledge organization;

- 2014, realizado em Cracóvia, na Polônia, com o tema Knowledge organization in the 21st century;

- 2016, realizado no Rio de Janeiro, no Brasil, com o tema Knowledge organization for a sustainable world.

É importante ressaltar que o objetivo da pesquisa é identificar os conceitos culturais por meio dos eventos realizados e não por meio das publicações realizadas. Embora a materialidade da pesquisa se revele por meio dos anais dos eventos, não será analisada a coleção completa Advances in Knowledge Organization - coleção essa que é composta pelos anais -, pois o volume 3 dessa coleção é constituído pelos trabalhos apresentados no Conferência ISKO Roma, este tendo características de capítulo regional da ISKO e não internacional.

Ressalta-se ainda, que embora a $2^{\circ}$ Conferência ISKO Internacional, realizada em Madras em 1992, possua em seus anais Prefácio e Introdução, a pesquisa teve acesso apenas à seção de Prefácio, sendo portanto, excluída a seção Introdução da presente análise. No $11^{\circ}$ evento, realizado em Roma em 2010, foi utilizado para análise o "Call for papers", seção presente no anais em substituição ao Prefácio e Introdução.

Por meio dessa análise, identificou-se enunciados que remetam ao contexto social da Organização do Conhecimento, fazendo ligações com os temas dos eventos, as linhas de pesquisa dos organizadores e apontou-se como ocorreu a sedimentação dessa temática no ambiente ISKO.

\section{RESULTADOS}

Antes de demonstrar como os aspectos sociais da ORC se construíram nos eventos da ISKO, é importante salientar que logo no primeiro anais, a instituição deixa bem claro o objetivo que consiste a promoção desses eventos: estabelecer contato entre os diversos pesquisadores em Organização e Representação do Conhecimento ao redor do mundo, buscando soluções para problemas da área. É sob essa premissa que os eventos se desenvolvem, de maneira que, ao longo do tempo, a instituição se consolidou enquanto a maior promotora dos estudos nesse campo, a nível internacional, como pôde ser identificado no decorrer da leitura do material de pesquisa.

Outro importante aspecto dessa primeira publicação é a ênfase na "transparência" nos trabalhos relacionados à organização do conhecimento, de forma a simplificar os processos de tratamento dessa informação para sua posterior recuperação. O editor, Dr. Robert Fugmann, afirma ser esse o grande conceito que pode ser identificado por meio dos trabalhos publicados nesse primeiro evento e que esse deve ser um requisito dos indexadores no momento de suas atividades.

É possível relacionar esse conceito de transparência com outros, como neutralidade, objetividade ou imparcialidade nos processos de organização, conceitos esses que foram demonstrados como difíceis de atingir em pesquisas recentes (GUIMARÃES, MILANI, EVANGELISTA, 2015; SILVA, 2016), pois as atividades de Organização e Representação do Conhecimento são realizadas em sua grande maioria por meios intelectuais, podendo portanto, sofrer influências dos profissionais que as realizam.

Após esse momento, questões relacionadas ao contexto social são apresentadas novamente em 1996, onde seções sobre questões multiculturais e multilinguísticas são apresentadas, demonstrando preocupações com o acesso de 
diferentes culturas e idiomas a uma mesma unidade informacional ou ferramenta de organização do conhecimento.

Depois, no $6^{\circ}$ Congresso ISKO Internacional, mais uma vez a preocupação com diferentes culturas e idiomas é apresentada, dessa vez, enquanto um subtema do evento denominado "Culture, Language and Communication in Knowledge Organization". O surgimento dessas noções em um nível mais alto, como o de um subtema, devese em grande parte pela influência das organizadoras do evento, em especial Dra. Clare Beghtol, que realizou importantes pesquisas relacionadas a garantia cultural (2002), ponderando sobre essas ferramentas serem traduzidas de forma mecânica, sem uma devida reflexão sobre os termos usados pela nova comunidade usuária.

No próximo congresso, as questões culturais começam a ficar ainda mais perceptíveis. Dessa vez, não apenas as questões multiculturais aparecem em dois subtemas ("Integration of knowledge in multicultural domain-oriented systems (part 1)" e "Integration of knowledge in multicultural domain-oriented systems (part 2)"), como também é criado um subtema relacionado às questões éticas do profissional da informação, denominado "Professional ethics, users and information structures". A organizadora desse capítulo, Dra. María José López-Huertas, ainda revela a importância desses subtemas pois a preocupação com as minorias tornamse cada vez mais frequentes. A consolidação desses assuntos em subtemas é relacionada ainda ao tema do evento, "Challenges in knowledge representation and organization for the 21st century", que se propõe a refletir sobre os desafios que os profissionais da informação deverão enfrentar no novo século. É nesse momento que as questões éticas e culturais dão seu primeiro passo de maneira mais evidente nas publicações das conferências ISKO Internacional.

Nas publicações posteriores, as temáticas relacionadas ao multiculturalismo e multilinguismo encontram-se mais consolidadas, sempre presentes nos subtemas dos congressos.

No décimo evento, é o primeiro momento que as questões culturais aparecem de forma explícita no tema: "Culture and Identity in
Knowledge Organization", refletindo não apenas sobre diferentes culturas que compõem uma unidade informação mas ainda, como essas culturas se identificam e são identificadas pelos sistemas de ORC. Essa escolha é justificada pelo organizador do evento, Dr. Joseph Tennis, por conta do país em que o evento foi realizado: Canadá, que é composto por uma grande diversidade cultural, resultado das colonizações francesa e alemã e ainda, pelas comunidades nativas que ali habitavam no início.

Em 2010, os organizadores da $11^{\circ}$ Conferência, alertam que pesquisas já identificavam que os sistemas de Organização do Conhecimento são afetados pelos contextos sociais, culturais e filosóficos das comunidades usuárias. Dessa forma, um olhar crítico para esse âmbito tornava-se cada vez mais necessário. Os organizadores ainda chamam a atenção que, em um mundo cada vez mais globalizado, não há como prever quem serão os usuários daquele sistema e que portanto, essas ferramentas não podem ser produzidas sem se atentar às diversidades.

Essa ideia de que diferentes culturas possuem seu impacto no desenvolvimento da ORC, é reforçada na $12^{\circ}$ Conferência ISKO Internacional.

A última Conferência foi realizada no ano de 2016, no Rio de Janeiro e expandiu ainda mais os aspectos culturais enquanto subtemas, incluindo ainda os aspectos sociais políticos da Organização e Representação do Conhecimento: educação e práticas profissionais, ética, cultura e identidade e desenvolvimento sustentável. $\mathrm{O}$ evento ainda teve uma mesa redonda, ministrada pela Dra. María José López-Huertas, sobre a influência da cultura nas atividades de ORC. Esse detalhamento dos subtemas e a expansão para uma discussão de mesa-redonda, pode ser justificada pelo organizador do evento, Dr. José Augusto Chaves Guimarães, ser um grande estudioso das questões éticas em Organização e Representação do Conhecimento (GUIMARÃES; FERNANDEZ-MOLINA, 2002; GUIMARÃES et al. 2008, GUIMARÃES; MILANI; EVANGELISTA, 2015).

Esses resultados podem ser melhor visualizados na linha do tempo a seguir. 
Figura 1: Linha do tempo da dimensão cultural na ISKO

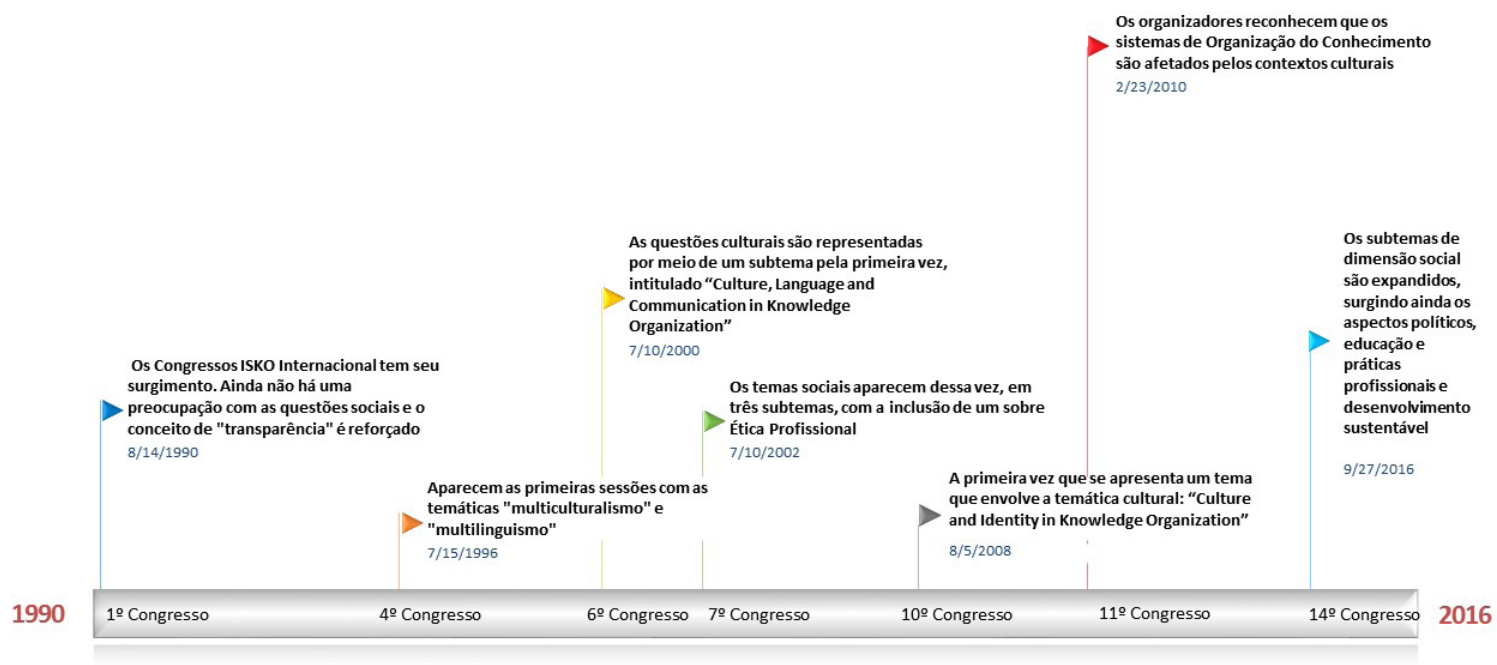

Fonte: Elaborada pelos autores

\section{CONSIDERAÇÕES FINAIS}

O presente artigo propôs fazer uma explanação sobre a metodologia de Análise do Discurso, buscando evidenciar seus principais teóricos, seus conceitos base e apresentar pesquisas no âmbito da Ciência da Informação. Buscou-se ainda utilizar dessa metodologia para analisar a construção da temática social e cultural no âmbito dos eventos internacionais promovidos pela International Society for Knowledge Organization.

Embora não seja possível chegar-se a conclusões definitivas a partir da análise realizada, uma vez que se focou apenas em um tipo de material (os editoriais dos anais dos eventos ISKO Internacional), foi possível chegar a algumas considerações interessantes e que podem instigar futuras pesquisas.

Embora a ISKO enquanto sociedade surge já estimada no campo da Organização e Representação do Conhecimento, percebe-se que as preocupações com suas questões sociais foram se construindo ao longo dos congressos, avançando cada ano para um novo nível de representatividade.
Levaram-se seis anos para que a temática fosse tratada pela primeira vez em um editorial, no qual houve o interesse em relação às diversas culturas e idiomas que podem se utilizar das ferramentas de ORC e das unidades informacionais como um todo. Dez anos depois, tópicos como ética, prática profissional, política, sustentabilidade, cultura e identidade aparecem não apenas como subtemas, mas dentro de toda uma dimensão do congresso para tratá-los.

Percebeu-se também que os organizadores influenciam em peso na proporção que esses temas são tratados. Os congressos que tiveram as questões culturais representadas em maior proporção, foram organizador por pesquisadores que trabalham com esses assuntos em suas pesquisas, como Clare Beghtol (2000), Maria José López-Huertas (2002), Joseph Tennis (2010) e José Augusto Chaves Guimarães (2016).

$\mathrm{O}$ artigo é finalizado com a reflexão de que, embora a tônica sociocultural não seja uma coisa que tem seu surgimento a partir da consolidação teórica da Organização e Representação do Conhecimento, ela vem se apresentando e ganhando importante espaço nas últimas pesquisas sobre o campo, demonstrando que não 
apenas é necessária para o desenvolvimento ético dos processos de organização, mas que também é fundamental que as pesquisas nesse sentido se façam cada vez mais presentes.
Agradecimento: A autora Isadora Victorino Evangelista agradece o auxílio recebido pela Fundação de Amparo à Pesquisa do Estado de São Paulo, processo 2017/02327-8.

Artigo recebido em 04/02/20I8 e aceito para publicação em 20/04/20I8

\title{
THE CULTURAL AND SOCIAL DIMENSION OF KNOWLEDGE ORGANIZATION: an discourse analysis of International Society for Knowledge Organization editorials
}

\begin{abstract}
Considering the improve of researches about cultural questions in Knowledge Organization (KO) and the International Society for Knowledge Organization (ISKO) as the most representative institution in studies about KO, queries how the cultural aspects in the area appears and was constructed in the international events of ISKO. To do so, we analyzed the concepts related to this thematic in the editorials published in the events publications. As methodology, was used the Foucault Discourse Analysis, studied by Pêcheux and his group, because it has as characteristic the ideologies rupture and worries in evidence the relations between dominant and dominated. As results, was possible to see that the cultural concepts appears only in the $4^{\text {th }}$ Congress, where was reflects a concern with multicultural and multilingual issues. After this first moment, these questions arise in subthemes, congress themes, round-tables and, in the last Congress, in a dimension dedicated to this universe. As conclusion, was possible to infer that this area of studies is in expansion in the last years, showing that it is not only necessary to the ethical developing of $K O$, but it is fundamental that researches in this sense increasingly.
\end{abstract}

Keyword: Knowledge Organization. Sociocultural aspects in KO. International Society for Knowledge Organization. Discourse Analysis.

\section{REFERÊNCIAS}

\author{
ALBRECHTSEN, H.; OERNAGER, S. Knowledge \\ Organization and quality management. \\ Frankfurt: Indeeks, 1994.
}

ARSENAULT, C.; TENNIS, J. T. (eds.). Cultural and Identity in Knowledge Organization. Würzburg: ERGON, 2008.

BABIK, W. (ed.). Knowledge organization in the 21st century. Würzburg: ERGON, 2014.

BARROS, T. H. B. Analise do discurso e a ciência da informação: implicações teóricas para a conjuntura do documento no Brasil. In: GUERRERO, Antonio Pulgarín; MORENO, Adustín Vivas (Org.). Límites, fronteras y espacios comunes: encuentros y desencuentros em lãs Ciencias de La Información. Badajoz: Ed. da Universidade de Extremadura, 2011, v. 1, p. 508-519.
Os Arquivos, A Arquivística e o Discurso: alguns marcos históricos e conceituais. Informação Arquivística, v. 2, p. 135-157, 2013.

A representação arquivística: uma análise do discurso teórico e institucional a partir dos contextos espanhol, canadense e brasileiro. 2015. Tese (Doutorado em Ciência da Informação), UNESP: Marília, 2014.

Por uma teoria do discurso: reconsiderações histórico-conceituais. In: Uma trajetória da Arquivística a partir da Análise do Discurso: inflexões histórico-conceituais. São Paulo: UNESP, 2015, p. 27-71.

BEGHTOL, C.; HOWARTH, L. C.; WILLIAMSON, N. J. (eds.). Dynamis and stability in knowledge organization. Wuzburg: Ergon, 2000.

BEGHTOL, C. A proposed ethical warrant for global knowledge representation and 
organization systems. Journal of Documentation, Londres, v. 58, n. 5, p. 507-532, 2002.

BUDIN, G.; SWERTZ, C.; MITGUTSCH, K. Knowledge Organization for a global learning society. Wuzburg: Ergon, 2006.

DAHLBERG, I. Ethics and knowledge organization: in memory of Dr. S. R. Ranganathan in his centenary year. International Classification, Frankfurt, v. 19, n. 1, p. 1-2,1992.

EL HADI, W. M.; MANIEZ, J.; POLLITT, S. (eds). Structures and relations in knowledge organization. Wuzburg: Ergon, 1998.

FREITAS, L. S. de. Sentidos da história e história dos sentidos da Ciência da Informação: um esboço arqueológico. Morpheus: Rio de Janeiro, n. 2, 2003.

Entre o público e o privado: trajetos temáticodiscursivos da área de informação. Informação \& Sociedade, João Pessoa, v. 14, n. 1, 2004.

A análise do discurso e o campo informacional: usos atuais e alcance epistemológico. InCID, Ribeirão Preto, v. 1, n. 1, p. 32-55, 2010.

FROHMANN, B. Discourse analysis as a research method in library and information Science. Library and Information Science Research, Perth, v. 16, p. 119-138, 1994.

Discourse and documentation: some implications for pedagogy and research. Journal of Education for Library and Information Science, Oak Ridge, v. 42, n. 1, 2001.

FUGMANN, Robert (ed.). Tools for knowledge organization and the human interface. Frankfurt: Indeks, 1990.

GARCÍA GUTIÉRREZ, A. Knowledge organization from a "culture of the border" towards a transcultural ethics of mediation. In: LÓPEZ-HUERTAS, M. M. (Ed.). Challenges in knowledge representation and organization for the 21st century: integration of knowledge across boundaries. Wüsburg: ERGON, 2002. p. 516-522.

GASPAR, N. R. Análise do discurso: a leitura no foco do audiovisual. Polifonia, v. 13, p. 59-76, 2008.
GASPAR, N. R.; REIS, L. de L. Um olhar da análise do discurso para a representação temática na Ciência da Informação. Datagramazero, Rio de Janeiro, v. 11, p. 01, 2010.

GNOLI, C.; MAZZOCCHI, F. Paradigms and conceptual systems in knowledge organization. Würzburg: ERGON, 2010.

GREEN, R. Knowledge organization and change. Frankfurt: Indeeks, 1996.

GREGOLIN, M. do R. Foucault e Pêcheux na análise do discurso: diálogos \& duelos. São Carlos: Claraluz, 2006.

GUIMARAES, J. A. C.; FERNÁNDEZ-MOLINA, J. C. Los aspectos éticos de la organización y representación del conocimiento en la revista Knowledge Organization. In: FRÍAS, J. A.; TRAVIESO, C. (Org.). Tendencias de investigación en organización del conocimiento. Salamanca: Universidad de Salamanca, 2002. p. 809-816.

GUIMARÃES, J. A. C. et al. Ethics in the knowledge organization environment: an overview of values and problems in the LIS literature. In: ARSENAULT, C.; TENNIS, J. T. (Ed.). Cultural and Identity in Knowledge Organization. Würzburg: ERGON Verlag, 2008. p. 340-346.

GUIMARÃES, J. A. C.; MILANI, S. de O.; EVANGELISTA, I. V. Valores éticos na organização e representação do conhecimento. Encontros Bibli, Florianópolis, v. 20, n. 1, p. 19 32, 2015.

GUIMARÃES, J. A. C.; MILANI, S. O.; DODEBEI, V. Knowledge Organization for a sustainable world. Würzburg: ERGON, 2016. (Advances in Knowledge Organization, 15).

HJØRLAND, B. Knowledge Organization (KO). Knowledge Organization, v. 43, n. 6, p. 475-484, 2016.

HUDON, M. Multilingual thesaurus construction: integrating the views of different cultures in one gateway to knowledge and concepts. Knowledge Organization, Würzburg, v. 24, n. 2, p. 84-91, 1997. 
LÓPEZ-HUERTAS, M. J. (ed.). Challenges in knowledge representation and organization for the 21st century. Wuzburg: Ergon, 2002.

Cultural impact on Knowledge Representation and Organization in a Subject Domain. In: ARSENAULT, C.; TENNIS, J. T. (Ed.). Cultural and Identity in Knowledge Organization. Würzburg: ERGON Verlag, 2008. p. 340-346.

MCILWAINE, I. C. Knowledge organization and the global information society. Wuzburg: Ergon, 2004.
MITTMANN, S. Discurso e texto: na pista de uma metodologia de análise. In: FERREIRA, Maria Cristina; INDURSKY, Freda. Análise do Discurso no Brasil: mapeando conceitos, confrontando limites. São Carlos: Claraluz, p. 153-161, 2007.

NEELAMEGHAN, A.; RAGHAVAN, K. S. (eds.). Categories, contexts and relations in knowledge organization. Würzburg: ERGON, 2012.

SILVA, A. P. Aspectos éticos em Organização da Informação: um estudo em códigos de ética do profissional arquivista. 2016. Dissertação (Mestrado em Ciência da Informação), UNESP: Marília, 2016. 